\title{
Haematological Changes in Tuberculosis with Special Reference to Iron Metabolism
}

\author{
Avneesh Malviya ${ }^{1}$, Neelima Bahal ${ }^{2}$ \\ 1Department of Pathology, Shri Guru Ram Rai Institute and Medical Sciences, Dehradun, Uttarakhand, India. \\ ${ }^{2}$ Department of Pathology, Shri Guru Ram Rai Institute and Medical Sciences, Dehradun, Uttarakhand, India.
}

\section{ABSTRACT}

\section{BACKGROUND}

Tuberculosis is an ancient human disease that has long been a major public health challenge in the world and remains a major health problem in most developing countries. Tuberculosis is uncommon in most parts of the western world, except for the geriatric population and in patients with AIDS, where it is assuming increasing importance. In the third world, however, it remains a major problem. In South Africa it is still very common and is a major cause of death.

\section{METHODS}

For the present case control study, 45 cases were selected from patients attending the OPD and admitted in the Department of Tuberculosis and Chest Disease, S.N. Medical College, Agra, during 2000 - 2003. 23 were male and 22 were female.

\section{RESULTS}

In the current study a total of 45 cases of pulmonary tuberculosis (sputum positive) were studied. 23 were males and 22 were females. The age ranged from 18 years to 65 years. 27 cases were evaluated prior to initiation of therapy whereas the remainder were evaluated 3 months after initiation of therapy of which 3 were evaluated after 4 months of therapy.

\section{CONCLUSIONS}

The short period of therapy did not result in a decline in the frequency of iron deficiency anaemia or anaemia of chronic disorders. Megaloblastosis was not encountered in any of the patients after therapy.

\section{KEY WORDS}

Tuberculosis, Infectious Disease, Hematologic Abnormalities, Iron Metabolism
Corresponding Author:

Dr. Avneesh Malviya,

Assistant Professor,

SGRR IH \& MS, Patel Nagar,

Dehradun-248001,

Uttarkhand, India.

E-mail: avneeshmalviya@gmail.com

DOI: $10.14260 / j e m d s / 2020 / 465$

How to Cite This Article:

Malviya A, Bahal N. Haematological changes in tuberculosis with special reference to iron metabolism. J. Evolution Med. Dent. Sci. 2020;9(30):2133-2138, DOI: $10.14260 /$ jemds/2020/465

Submission 22-11-2019,

Peer Review 14-06-2020,

Acceptance 22-06-2020,

Published 27-07-2020.

Copyright (c) 2020 JEMDS. This is an open access article distributed under Creative Commons Attribution License [Attribution 4.0 International (CC BY 4.0)] 


\section{BACKGROUND}

Tuberculosis is an ancient human disease that has long been a major public health challenge in the world and remains a major health problem in most developing countries. Evidence of existence of tuberculosis has been found in the bones of prehistoric man.[1,2] These remains date back to about 8000 B.C. ${ }^{[3]}$ The theory that tuberculosis is an infectious disease was conceived by Aristotle more than 2000 years before that discovery of tubercle bacillus.[4] The issue was finally settled by Villemin who demonstrated in a series of classical experiments that tuberculosis is caused by a special agent and that it can be transmitted from man to animals by inoculation of infected material. [5,6] At last Rebert Koch discovered tubercle bacilli in .[3,7]

Tuberculosis is uncommon in most parts of the western world, except for the geriatric population and in patients with AIDS, it is assuming increasing importance. In the third world, however, it remains a major problem. In South Africa it is still very common and is a major cause of death. In 1992, WHO published estimates of prevalence of tuberculosis infection and incidence of disease. The prevalence of tuberculous infection was estimated to be 1.7 billion persons or approximately one third of the world population. The annual incidence of new cases of tuberculosis was estimated to be slightly more than 8 million patients. Published estimates by the WHO of the number of new cases of tuberculosis expected in $1995(8,768,000)$ and $2000(10,222,000)$ indicate a steadily growing problem. $95 \%$ of all the cases reside in South-East Asia, Western pacific and Africa.[8]

Hematologic abnormalities have been described in association with mycobacterial infection for almost 100 years. Patients with both pulmonary and extra pulmonary tuberculosis may demonstrate peripheral blood abnormalities and the severity of the findings may be minimal or profound. Hematologic changes associated with pulmonary tuberculosis have been incompletely investigated apart for two reports till 1989.[4] The findings in miliary tuberculosis have been reasonably well documented. Other studies have reported the haematological changes in pulmonary tuberculosis in limited details or as information incidental to main object of the investigation ${ }^{5}$. Investigators have repeatedly addressed the possible casual relationships between blood abnormalities and mycobacterial infection.

Reversible peripheral blood abnormalities are commonly associated with pulmonary tuberculosis, but whether disseminated tuberculosis or atypical disease can cause profound bone marrow and peripheral blood abnormalities by modulating normal haematopoiesis remains controversial. The relationship between profound blood abnormalities and mycobacterial infection has come from an understanding of the immunology of mycobacterial infection ${ }^{9}$. The only convincing proof that tuberculosis causes a given hematologic abnormality would be the documentation of its disappearance with successful antituberculous therapy alone.

There are four possible relationship of tuberculosis to hematologic disease. these are-

1. The hematologic disease predisposes to tuberculosis reactivation.
2. Tuberculosis causes or is associated with hematologic abnormalities.

3. Antituberculous drugs cause hematologic abnormalities.

4. The hematologic disease and tuberculosis are coincidental.

The anaemia of chronic inflammation is associated with several disturbances of iron metabolism.[6] These include hypoferremia, hypotransferrinaemia, a reduced percentage saturation of transferrin, a raised plasma ferritin and increased reticuloendothelial iron stores. In addition, there is evidence that several of these changes (including the hypoferremia, tissue redistribution of iron and raised serum ferritin) may occur as a part of the acute phase response and are mediated by interleukin-1.[10] The factors which have been identified as contributing to the anaemia itself include a decreased red cell survival, a reduced erythropoietin response and iron deficient erythropoiesis secondary to the reduced percentage saturation of transferrin.[11] The relative importance of this last mechanism has, however, been questioned.

Hypochromic anaemia associated with the accumulation of excessive numbers of iron granules in the perinuclear zone of erythroblasts ("Sideroblastic Anaemia") has been described in patients being treated with isoniazid [12]. The condition is apparently very rare in patients receiving isoniazid. However, sideroblastic anaemia does not appear to be infrequent in patients given isoniazid in conjunction with other antituberculous drugs such as cycloserine or pyrazinamide. With this knowledge, we are going to study the haematological changes and iron status associated with tuberculosis.

\section{Objectives}

1. To assess haematological changes in tuberculosis patients.

2. To assess serum iron level in tuberculosis patients.

3. To evaluate bone marrow iron in aspirates from tuberculosis patients.

\section{METHODS}

For the present case control study, 45 cases were selected from patients attending the OPD and admitted in Department of Tuberculosis and Chest disease, S.N. Medical College, Agra during 2000-2003. 23 were male and 22 were female. The sample size was taken based on the convenience of the study. The age ranged from 18 years to 65 years 27 cases were evaluated prior to initiation of therapy whereas, the remainder were evaluated 3 or more months after therapy. We had also studied 15 controls, of which 8 were male. The control cases were evaluated for haemoglobin, peripheral blood changes, ESR, platelet count and serum iron. The study was approved by Ethics committee and informed consent was obtained.

\section{Selection of Patients}

Only sputum positive cases were evaluated in the study. 


\section{Sample}

Blood sample is collected in E.D.T.A vial and plain vial along with bone marrow aspiration was done

\section{Assay}

Following investigations were done to assess the haematological changes in those patients.

1. Haemoglobin estimation by cyanmethemoglobin method (Dacie and Lewis 1991).

2. TLC with Turk's fluid with help of Neubauer chamber.

3. DLC peripheral blood smear stained by Leishman stain.

4. Platelet count - with $10 \mathrm{~g} / \mathrm{l}$ ammonium oxalate solution as diluents with the help of Neubauer chamber.

5. ESR by Wintrobe's method.

6. Serum iron level with the help of commercially available kits (Ferrozine method)

7. Bone marrow examination - Bone marrow aspirate smears stained by Romanowsky method.

8. Perl's reagent to assess bone marrow iron.

\section{Statistical Methods}

The statistical package for Social Science \{SPSS\} Version 20 will be used for Data Analysis. Mean, median, and SD are used to describe quantitative data. Qualitative data are summarized using frequency and percentage.

\section{RESULTS}

In the current study a total of 45 cases of pulmonary tuberculosis (sputum positive) were studied. 23 were males and 22 were females. The age ranged from 18 years to 65 years 27 cases were evaluated prior to initiation of therapy whereas the remainder were evaluated 3 months after initiation of therapy of which 3 were evaluated after 4 months of therapy.

\begin{tabular}{|c|c|c|}
\hline & Patients (27) & Controls (15) \\
\hline Anaemia of chronic disorder & $12(48 \%)$ & $11(64.7 \%)$ \\
\hline Iron deficiency anaemia & $4(16 \%)$ & $3(17.6 \%)$ \\
\hline Megaloblastic anaemia & $3(12 \%)$ & $0(0 \%)$ \\
\hline Normal haemoglobin & $0(0 \%)$ & $3(17.64 \%)^{*}$ \\
\hline Unexplained anaemia & $6(24 \%)$ & $0(0 \%)$ \\
\hline Bone marrow not suitable for iron assessment & 2 & 2 \\
\hline Total & 27 & 18 \\
\hline \multicolumn{3}{|c|}{ Table 1. Evaluation of 27 Untreated \& 18 Treated Cases } \\
\hline \multicolumn{3}{|c|}{ *(includes one cases of evolving anaemia of chronic disorders) } \\
\hline
\end{tabular}

In the current study 27 patients were studied before initiation of therapy, 18 patients were studied after the therapy. Anaemia of chronic disorder was found in 23 patients (54.76\%), 12 (48\%) patients in untreated \& 11 (64.11\%) in treated group.

Iron deficiency anaemia was found in 7 patients (16.66\%), $4(16 \%)$ in untreated and $3(17.64 \%)$ in the treated group.

Megaloblastic anaemia was found only in $3(12 \%)$ cases in untreated patients, overall frequency was $7.14 \%$. In untreated group $6(24 \%)$ cases were found with unexplained anaemia. $3(17.64 \%)$ cases had normal haemoglobin in treated group in which one case was evolving anaemia of chronic disorder. 3 bone-marrow aspirates were not suitable for assessment of iron ( 2 in untreated, 1 in treated group).

\begin{tabular}{|ccc|}
\hline Haemoglobin Evaluation & Patients (27) & Controls (15) \\
Non Anaemic & $0(0 \%)$ & $5(33.33 \%)$ \\
Mild Anaemia & $14(51.85 \%)$ & $10(66.66 \%)$ \\
Moderate Anaemia(Hb-6-9 g/dL) & $11(40.74 \%)$ & $0(0 \%)$ \\
Severe Anaemia(Hb $<6 \mathrm{~g} / \mathrm{dL})$ & $2(7.40 \%)$ & $0(0 \%)$ \\
Range & $2.5-12.5 \mathrm{~g} / \mathrm{dL}$ & $11-14 \mathrm{gm} / \mathrm{dL}$ \\
Mean & $8.8 \mathrm{gm} / \mathrm{dL}$ & $12.06 \mathrm{gm} / \mathrm{dL}$ \\
\hline Table 2. Haemoglobin Evaluation in 27 \\
Untreated Patients and 15 Controls \\
\hline
\end{tabular}

Haemoglobin range from $2.5 \mathrm{gm} / \mathrm{dL}$ (control $11 \mathrm{~g} / \mathrm{dL}$ ) to $12.5 \mathrm{gm} / \mathrm{dL}$ (control $14 \mathrm{gm} / \mathrm{dL}$ ) at the time of diagnosis, with a mean of $8.81 \mathrm{gm} / \mathrm{dL}$ (control $12.06 \mathrm{gm} / \mathrm{dL}$ ). There was no case of non-anaemic (control 33.33\%). Of the anaemic group, $51.85 \%$ had mild anaemia (control 66.66\%), 40.74\% had moderate anaemia (control $0 \%$ ), $7.40 \%$ had severe anaemia (control 0\%). Thus $100 \%$ of cases were anaemic (control $66.66 \%)$.

\begin{tabular}{|cc|}
\hline Haemoglobin Evaluation & Patients (18) \\
Non Anaemic & $3(16.66 \%)$ \\
Mild Anaemia & $11(61.11 \%)$ \\
Moderate Anaemia (Hb-6-9 g/dL) & $4(22.22 \%)$ \\
Severe Anaemia $(\mathrm{Hb}<6 \mathrm{~g} / \mathrm{dL})$ & $0(0 \%)$ \\
Range & $6.5-13 \mathrm{~g} / \mathrm{dL}$ \\
Mean & $10.30 \mathrm{~g} / \mathrm{dL}$ \\
\hline Table 3. Haemoglobin Evaluation in 18 Treated Patients \\
\hline
\end{tabular}

Haemoglobin in 18 treated patients ranged from 6.5 $\mathrm{gm} / \mathrm{dL}$ to $13 \mathrm{gm} / \mathrm{dL}$ (untreated 2.5-12.5 gm/dL) after 3 months of initiation of therapy with a mean of $10.30 \mathrm{gm} / \mathrm{dL}$ (untreated $8.81 \mathrm{~g} / \mathrm{dL}$ ). $16.66 \%$ cases (3 cases) were nonanaemic, of which 2 cases were female. The severity of anaemia was characterized as mild in $61.11 \%$ (untreated$57.85 \%$ ), moderate in $22.22 \%$ (untreated $40.74 \%$ ), severe anaemia was not seen in any patients (untreated $7.40 \%$ ). Thus 83.33\% of cases were anaemic (untreated $100 \%$ ).

\begin{tabular}{|c|c|c|}
\hline & Patients (27) & Controls (15) \\
\hline Non Anaemic & $0(00 \%)$ & \\
\hline Normocytic Normochromic & & $5(33.33 \%)$ \\
\hline Anaemia. & $27(100 \%)$ & \\
\hline Normocytic Normochromic & $19(70.3 \%)$ & $10(66.66 \%)$ \\
\hline Microcytic Hypochromic & $4(14.81 \%)$ & \\
\hline Normocytic Hypochromic & $1(3.7 \%)$ & - \\
\hline Microcytic Normochromic & $0(0 \%)$ & \\
\hline Macrocytic Normochromic & $3(11.1 \%)$ & \\
\hline Macrocytic Hypochromic & $0(0 \%)$ & \\
\hline \multicolumn{3}{|c|}{$\begin{array}{c}\text { Table 4. Evaluation of R.B.C. Morphology in } \\
27 \text { Untreated Patients and } 15 \text { Controls }\end{array}$} \\
\hline
\end{tabular}

All the 27 patients were anaemic at the time of diagnosis. The Red Blood Cells were characterized as normocytic normochromic in $70.37 \%$ (100\% in both anaemic and nonanaemic control group). $14.81 \%$ were microcytic hypochromic, $11.11 \%$ were macrocytic normochromic and rest $3.70 \%$ were normocytic hypochromic.

\begin{tabular}{|cc|}
\hline Non Anaemic & Patients (18) \\
Normocytic Normochromic & $3(16.66 \%)$ \\
Anaemia. & $3(100 \%)$ \\
Normocytic Normochromic & $15(83.33 \%)$ \\
Microcytic Hypochromic & $13(86.66 \%)$ \\
Normocytic Hypochromic & $1(6.66 \%)$ \\
Microcytic Normochromic & $0(0.0 \%)$ \\
Macrocytic Normochromic & $0(0.0 \%)$ \\
Macrocytic Hypochromic & $1(6.66 \%)$ \\
\hline Table 5. Evaluation of R.B.C. Morphology in 18 Treated Patients \\
\hline
\end{tabular}


After the therapy $3(16.66 \%)$ patients were non-anaemic, and Red Blood Cells in all (100\%) patients were characterized as normocytic normochromic. 15 (83.33\%) cases were anaemic, Red Blood Cells in them characterized as normocytic normochromic in $86.66 \%$ (untreated $70.31 \%$ ), microcytic hypochromic in $6.66 \%$ (untreated $14.81 \%$ ) and macrocytic normochromic in $6.66 \%$ (untreated $11.11 \%$ ). Thus normocytic normochromic was $88.88 \%$ of cases after therapy.

\begin{tabular}{|ccc|}
\hline & Patients (27) & Controls (15) \\
Normal & $13(48.14 \%)$ & $15(100 \%)$ \\
Leukocytosis & $12(44.44 \%)$ & $0(0 \%)$ \\
Leukopenia & $2(7.40 \%)$ & $0(0 \%)$ \\
Range & $1500-21,200 /$ cumm & $4200-8,800 /$ cumm \\
Mean & $9,785.18 /$ cumm & $6.213 .33 /$ cumm \\
\hline Table 6. Evaluation of Total Leukocyte Count \\
in 27 Untreated Patients \& 15 Controls \\
\hline
\end{tabular}

Total leukocytes count ranged from 1,500/cumm (control $4,200 /$ cumm) to $21,200 /$ cumm (control $8,800 /$ cumm) at the time of diagnosis, with a mean of $9,785.15 /$ cumm (control $6,213.33$ /cumm). Leukocytosis was $44.44 \%$, Leukopenia in $7.40 \%$ and normal count in $48.14 \%$ of cases (control $100 \%$ ). In patients with leukocytosis, $8(66.66 \%)$ cases were in range of $10,000-15,000 /$ cumm, 3 (25\%) cases were in range of $15,000-20,000 /$ cumm and $1(8.33 \%)$ case had more than $20,000 /$ cumm count. 2 cases had leukopenia, one had count $3,500 /$ cumm and other has count 1,500/cumm. 2 cases with leukopenia were pancytopenic.

\section{DISCUSSION}

In the current study a total of 45 cases of pulmonary tuberculosis were studied, 23 were male and 22 were female. The age ranged from 18 years to 65 years. 27 cases were evaluated prior to initiation of therapy whereas the remainder were evaluated 2 months after initiation of therapy of which 3 were evaluated after 4 months of therapy. We had also studied 15 control; of which 8 were male.

In the current study, on the basis of peripheral blood, serum iron and bone marrow findings, in pre-therapy group of cases, anaemia of chronic disorders, iron deficiency anaemia and megaloblastic anaemia was found in $48 \%, 16 \%$ and $12 \%$ of cases respectively. In 6 cases (24\%), cause of anaemia could not be categorized, possible etiological causes include endocrinopathy, evolving anaemia of chronic disorder and technical cause for this unspecified anaemias. Cameron et al(8) found anaemia of chronic disorder, iron deficiency and megaloblastic anaemia in $26.5 \%, 38.77 \%$ and $6.12 \%$ of their cases respectively. Post therapy group of current study revealed anaemia of chronic disorders, iron deficiency anaemia in $64.7 \%$ and $17.64 \%$ of cases respectively. Megaloblastic anaemia was not detected in post therapy group. In 2 cases of iron deficiency anaemic with normal S. iron was a surprise finding lending itself to possible interpretation of concomitant iron therapy instituted to patients. Haemoglobin was in the normal range in 3 cases of post therapy group including 1 case of resolving anaemia of chronic disorders.

In the current study, in the untreated group, mild to moderate anaemia was common but severe anaemia was rarely found ( 2 out of 27 untreated cases) in patients with pulmonary tuberculosis. Mild anaemia was also common in control group. This is also suggested by Charles D.W. Morris et al (13) and Arthur R. Bird (14) in their studies they noted anaemia in $66.5 \%$ and $58.5 \%$ (male $72 \%$, female $45 \%$ ) of their cases respectively. In the current study after therapy, mild anaemia and moderate anaemia was $61.11 \%$ and $22.2 \%$ respectively, no patients had severe anaemia, Anaemia was not present in $16.66 \%$ of cases. Daphne H. Line (1970) and S.J. Cameron(8), noted anaemia in $23 \%$ and $20 \%$ of their cases after therapy.

Majority (70.37\%) of patients in this series had normocytic normochromic red cells before initiation of therapy. Arthur R. Bird et al, observed 95\% normocytic normochromic blood picture, their findings are consistent with this current study. C.D.W. Morris, observed normocytic normochromic red cells in $66 \%$ of their cases. In the current study microcytic hypochromic and macrocytic normochromic red cell morphology was observed in $14.81 \%$ and $11.11 \%$ of the untreated cases respectively, Arthur R. Bird et al(13), observed microcytosis in $14 \%$ of their cases, macrocytosis in $18 \%$ of their cases. Their findings are almost similar to the observation in this study. In the current study after therapy normocytic normochromic red cells were in $88.88 \%$ of cases, while microcytic hypochromic and macrocytic normochromic red cells were in $6.66 \%$ of cases each. The finding can be comparable to the finding before the therapy, normocytic normochromic red cells were increased after therapy, while frequency of microcytic hypochromic and macrocytic normochromic fallen down after therapy, indicating red blood cells moved towards normality after therapy.

In this study, Leukocytosis was observed in $44.44 \%$ of untreated cases, all were due to neutrophilia, although one case had associated monocytosis and two cases had associated monocytosis and eosinophilia. Arthur R Bird, observed leucocytosis in $40 \%$ of patients. Daphne H. Line, found a mean total leucocyte count of $10,000 /$ cumm in their patients with anaemia, which is similar to that observed in current study (9,785/cumm), CDW Morris, noted leukocytosis in 55\% of their cases.

Leukopenia was detected in 2 cases $(7.40 \%)$ both pancytopenic, both were due to neutropenia and lymphopenia, S.J. Cameron (5), have found $5 \%$ of the cases with leukopenia all were due to neutropenia. This correlates with the current study. After the 3 months of therapy, the frequency of leucocytosis fell to $22.22 \%$ with a mean total leukocyte count of $9,127 /$ cumm. Daphne H. Line also observed a fall of mean total leucocyte count from 10,000 /cumm to 9,000 /cumm after therapy. This correlates with the findings in the current study.

In the differential leukocyte count neutrophilia was observed in $59.25 \%$ of untreated cases in the current study. Arthur R. Bird et al , observed neutrophilia in $57 \%$ of cases. CDW Morris, observed neutrophilia in $69 \%$ of cases the above two findings are consistent with the current findings. The frequency of neutropenia in the current study (11.11\%) was about twice as high as observed by S.J. Cameron (5\%). In the current study, lymphopenia was observed in $55.55 \%$ of untreated cases. CDW Morris observed lymphopenia in $22 \%$ of their cases. Arthur R Bird et al , reported lymphocytopenia in $17 \%$ of cases. Monocytopenia was observed in $37.03 \%$ of untreated cases in the current study, the corresponding figures in the series of Morris(14) and Bird(13) were 37\% and $50 \%$ respectively.

Eosinophilia was present in $14.81 \%$ of untreated patients in the current series. No mention of this could be discovered 
on reviewing the literature. Since about $6 \%$ of normal controls also revealed eosinophilia, too much importance cannot be attached to this observation. In the current study a mean of 3.07 lakh/cumm of platelet count was observed after the therapy. Thrombocytosis was observed in only $5.55 \%$ (1 case) which was much lower than before initiation of therapy (59.25\%), R.D. Baynes et al (2) observed mean platelet count as 2.83 lakh/cumm after therapy, Arthur R Bird et al, observed a mean of $3.26 \mathrm{lakh} /$ cumm in acid fast bacilli negative cases after the therapy.

In the current study, ESR (Wintrobe) ranged from 20-80 $\mathrm{mm} 1^{\text {st }}$ hour with a mean of $40.29 \mathrm{~mm} \mathrm{1st}$ hour with an increased ESR being observed in all the patients. Whereas it was found to be normal in all the control subjects. Arthur $\mathrm{R}$ Bird et al, observed a mean ESR of $74 \mathrm{~mm}$ 1st hour with increased ESR being noted in $80 \%$ of cases. CDW Morris (14), observed a mean ESR of $71 \mathrm{~mm}$ 1st hour with increased ESR being observed in $90 \%$ of cases. After the therapy ESR had fallen to a mean of $9 \mathrm{~mm}$ 1st hour, all the cases fell below 15 $\mathrm{mm} 1$ st hour, with an increased ESR being encountered in only $22.22 \%$ of cases and normal in $77.77 \%$ of cases. Daphne $\mathrm{H}$. Line et al, similarly observed ESR less than $15 \mathrm{~mm} 1 \mathrm{st}$ hour in all patients after the therapy.

In the current study Bone-Marrow examination was done on 27 patients before initiation of therapy. Plasmacytosis was noted in $29.62 \%$ of Bone-Marrow aspirates. S.J. Cameron (5), noted prominence of mature plasma cells in $17 \%$ of Bone Marrow Aspirates. Arthur R. Brid et al, observed plasmacytosis in $51 \%$ of bone marrow aspirates.

Increased eosinophils were noted in $11.11 \%$ of BoneMarrow aspirates before therapy, which is consistent with the frequency of eosinophilia (14.81\%) in the peripheral blood. Increased thrombopoiesis was observed in $22.22 \%$ of bone marrow aspirates before therapy, which somewhat correlates with increased platelet count (59.25\%). Erythroid hyperplasia was noted in $48.14 \%$ of patient before therapy which correlates with decreased M:E ratio (48.14\%).

Myeloid hyperplasia was noted in $11.11 \%$ of cases which correlates with increased M:E ratio (11.11\%). No specific mention of increased erythropoiesis, leucopoiesis or thrombopoiesis in the bone marrow aspirates, was discovered in the literature. After the therapy, Bone-marrow aspiration was done on 17 patients. Plasmacytosis was observed in $5.88 \%$ ( 1 case), much lower than findings at the time of diagnosis (29.62\%).

Increased eosinophils were observed in $17.64 \%$ of BoneMarrow aspirated after therapy, which was slightly higher than that of the time of diagnosis, this also supports the increased frequency of eosinophilia after the therapy in peripheral blood in comparison to eosinophilia at the time of diagnosis. After the treatment all the cases had normal thrombopoiesis. Before the therapy there were normoblastic erythropoiesis in $66.66 \%$, micronormoblastic erythropoiesis in $22.22 \%$ and megaloblastic erythropoiesis in $11.11 \%$. in one case erythropoiesis was normoblastic in which leucopoiesis showed megaloblastic changes. Daphne H Line et al (1970), found megaloblastosis at much higher frequency $(55.17 \%)$ in their series. However, this was not similar to the experience of other workers who noted megaloblastosis at much lower frequency $6 \%$ by S.J. Cameron $13.51 \%$ by Arthur R. Bird and $29.41 \%$ by Roberts et al (15). After the therapy, there were $88.23 \%$ normoblastic erythropoiesis and $11.76 \%$ had micronormoblastic erythropoiesis; thus the proportion of micronormoblastic marrow fell to about half \& megaloblastic marrow were not observed.

In the current study, storage iron was evaluated in 25 patients at the time of diagnosis, 2 marrows were not suitable for assessment of storage iron. Normal stores were observed in $72 \%$ of Bone-marrows, this is similar to the frequency of $70 \%$ reported by Daphne $\mathrm{H}$. Line et al(16) but much higher than the frequency of normal iron stores (17\%) reported by Cameron et al. Iron absent from store were in 20\% and increased in $8 \%$ of marrows of current untreated patients. As reported in the literature the frequency of absent iron stores ranges from $30 \%$ and Roberts et al (15) to $40 \%$. The reason for slightly lower frequency of absent storage iron in the untreated study is not known. Most marrows had store of grade $2+(48 \%)$ in the current study. In the current study of untreated patient the frequency of sideroblast was range from $2-38 \%$ with a mean of $13.24 \%$, reduced frequency were noted in $88 \%$ the corresponding figure in Daphne H. Line series was $65 \%$ which is slightly lower.

In the current study 17 patients were evaluated for storage iron after the therapy. Iron was absent from stores in $17.64 \%$ (untreated 20\%), normal stores were in 52.94\% (untreated $72 \%$ ) and increased stores in $29.14 \%$ (untreated 8\%). Daphne H. Line(16), observed mild increase in frequency of absence of storage iron after therapy. In the current series, the increased frequency of excessive storage iron after the therapy was an unexpected finding. It could possibly been a consequence of concomitant iron therapy which the patients may have taken. Other workers have noted increased frequency of sideroblasts after therapy (Robert et al, Daphne H Line) (16), this increase was also observed in the current study.

\section{CONCLUSIONS}

Based on peripheral blood, serum iron and bone marrow findings, anaemia of chronic disorders was detected in 23 $(54.76 \%)$ cases, iron deficiency anaemia in 7 (16.66\%) cases, megaloblastic anaemia in $3(7.14 \%)$ cases. 3 (17.645) cases restored to normal haemoglobin after therapy including one with resolving anaemia of chronic disorders. In six cases, $(14.28 \%)$, anaemia could not be clearly categorized on the basis of peripheral smear, serum iron and iron studies. The short period of therapy did not result in a decline in the frequency of iron deficiency anaemia or anaemia of chronic disorders. Megaloblastosis was not encountered in any of the patients after therapy.

Financial or Other Competing Interests: None.

\section{REFERENCES}

[1] Baynes RD, Bothwell TH, Flax H. Reactive thrombocytosis in pulmonary tuberculosis. J Clin Pathol 1987;40(6):6769.

[2] Bynes RD, Flax H, Bothwell TH. Haematological and iron related measurements in active pulmonary tuberculosis. Scand J Haematol 1986;36(3):280-7. 
[3] Biehl JP. Miliary tuberculosis: a review of Sixty-eight adult patients admitted to a Municipal General Hospital. Am Rev Tuberc 1958;77(4):605-22.

[4] Cavill I, Bentley DP. Erythropoiesis in the anaemia of rheumatoid arthritis. Br J Haematol 1982;50(4):583-90.

[5] Cavill I, Ricketts C, Napier JA. Erythropoiesis in the anaemia of chronic disease. Scant J Haematol 1977;19(5):509-12.

[6] Cobuen RJ, England JM, Samson DM, et al. Tuberculosis and blood disorder. Br J Haematol 1973;25(6):793-9.

[7] Cameron SJ, Horne NW. The effect of tuberculosis and its treatment on erythropoiesis and folate activity. Tubercle 1971;52(1):37-48.

[8] Cameron SJ. Tuberculosis and the blood - a special relationship? Tubercle 1974;55(1):55-72.

[9] Coley WB, Ewing J. Acute lymphatic tuberculosis with purpura haemorhagica. Trans Assoc Am Phys 1911;26:178.

[10] Weiss G. Iron and anaemia of chronic disease. Kidney International 1999;55(Suppl 69):S12-S17.
[11] Dannenberg AM Jr. Delayed-type hypersensitivity in all mediated immunity in pathogenesis of tuberculosis. Immunol Today 1991;12(7):228-33.

[12] Corr WP Jr, Kyle RA, Bowie EJ. Hematologic changes in tuberculosis. Am J Med Sci 1964;248(6):709-14.

[13] Morris CD. Disease profiles for white and black adult and geriatric patients. An analysis of 2,008 hospital medical admissions. S Afr Med J 1989;75(4):171-4.

[14] Morris CD, Bird AR, Nell H. The hematological and biochemical changes in severe tuberculosis. Q J Med 1989;73(272):1151-9.

[15] Roberts PD, Hoffbrand AV, Mollin DL. Iron and folate metabolism in Tuberculosis. Br Med J 1966;2(5507):198202.

[16] Line DH, Scintanidis B, Morgan JO, et al. The effect of chemotherapy of iron, folate and Vitamin B12, metabolism in tuberculosis. Q J Med 1971;40(159):33140. 